\title{
GRAMÍNEAS NATIVAS EN SUELOS DESNUDOS AL BORDE DE UNA CARRETERA Y SU SIMBIOSIS CON MICORRIZAS
}

\section{NATIVE GRASS SPECIES IN NAKED SOIL BORDERING A HIGHWAY AND THEIR SYMBIOSIS WITH MYCORRHIZAE}

\author{
Leónides Castellanos González ${ }^{1}$, Jesús Forero Cuadros ${ }^{2}$, Francisco Rodríguez Rincón ${ }^{3}$, Luis Roberto Sánchez Montano ${ }^{4}$
}

${ }^{1}$ Profesor, Ph.D., Facultad de Ciencias Agrarias. Unipamplona, Carretera a Bucaramanga km 1. Pamplona - Norte de Santander, Colombia, e-mail: leonides.castellanos@unipamplona.edu.co; ${ }^{2}$ Estudiante Ing. Agronómica, Facultad de Ciencias Agrarias. Unipamplona, Carretera a Bucaramanga km 1. Pamplona - Norte de Santander, Colombia, e-mail: jesusforeroc@ hotmail.com; ${ }^{3}$ Profesor, PhD., Facultad de Ciencias Básicas. Unipamplona, Carretera a Bucaramanga km 1. Pamplona - Norte de Santander, Colombia, e-mail: francisco.rodriguez@unipamplona.edu.co; ${ }^{4}$ Profesor, MSc., Facultad de Ciencias Básicas. Unipamplona. Carretera a Bucaramanga km 1. Pamplona - Norte de Santander, Colombia, e-mail: Irsanchez@unipamplona. edu.co

\author{
Rev. U.D.C.A Act. \& Div. Cient. 21(1): 253-257, Enero Junio, 2018 \\ https://doi.org/10.31910/rudca.v21.n1.2018.684
}

\section{INTRODUCCIÓN}

La construcción o la reparación de carreteras y de redes viales implica una intervención del paisaje, con el deterioro de los ecosistemas naturales, por lo que se impone tomar acciones para minimizar los impactos negativos, que esto conlleva. En varios países de Latinoamérica, existen programas de conservación de la red vial, que establece las regulaciones para evitar la erosión y la reposición de áreas verdes y céspedes, en las orillas de la carretera (Ministerio de Desarrollo Económico, 2002).

Los céspedes son muy utilizados para taludes en carreteras y la protección de las cárcavas, pudiéndose emplear como fajas o barreras costales (Tormo et al. 2009). Aunque se han desarrollado en los últimos años otros métodos para evitar la erosión, la restauración de la vegetación con gramíneas constituye un método importante y efectivo, en tiempo y en costos o mejorando el paisaje y protegiendo el medio ambiente (Díaz, 2011).

Para el establecimiento de los céspedes es importante tener en consideración las características físicas, químicas y biológicas del suelo. La importancia del suelo, para la productividad primaria, ha sido ampliamente documentada, por ser una fuente de agua y de elementos nutritivos, proveer, además, la matriz necesaria para diversos procesos biológicos, involucrados en el ciclo de nutrientes. El rol de las plantas en interacción con los microrganismos en la formación del suelo, particularmente la rizosfera, constituye un eslabón de estrecha dependencia y altamente sensibles a la perturbación.
El sustrato a colonizar debe reunir los requisitos nutricionales para las plantas y, de ser necesario, incorporar abonos orgánicos, como la turba u otros (Saldias, 2011). Tácuna et al. (2015) hacen referencia a la necesidad de incorporar materia orgánica para recuperar praderas y, de ser posible, emplear especies nativas, en programas de restauración ecológica.

La gramínea más utilizada para la restauración de la vegetación de taludes a los bordes de las carreteras es la especie introducida Brachiaria decumbens Stapf (Nufarm, 2016), pero su utilización implica gastos importantes en semilla y aplicación de materia orgánica.

En una evaluación de la diversidad microbiológica en suelos, se hace imprescindible investigar el rol funcional de los organismos clave, con el fin de definir el significado de la diversidad microbiana y los procesos relevantes en el ecosistema (Kjoller et al. 2000). El papel de las micorrizas en la rizosfera de las plantas favorece la nutrición mineral, principalmente, en cuatro aspectos: fisiología y desarrollo de la planta, crecimiento y morfología de raíces, procesos de absorción y disponibilidad de los nutrientes para las plantas (Medina \& Azcón, 2010).

Actualmente, se está construyendo una carretera desde Bosconia hasta San Roque, en el Departamento del Cesar, en Colombia y existen muchos espacios aledaños a la red vial, con suelos desnudos, donde se ha retirado la cubierta vegetal y se ha construido un terraplén. En el área señalada, se observaban especies de gramíneas que sobrevivían en esas condiciones extremas; entonces, surge la necesidad de identificarlas y de determinar la existencia o no simbiosis con hongos micorrizógenos arbusculares (HMA). 
El presente estudio de caso tuvo como objetivo determinar las especies de gramíneas presentes en suelos desnudos, aledaños a la carretera de Bosconia a Cuatro Vientos y la presencia de HMA en simbiosis.

\section{MATERIALES Y MÉTODOS}

Se realizó un recorrido de alrededor de $1,5 \mathrm{~km}$ en el terraplén aledaño a la carretera en construcción, entre Bosconia y Cuatro Vientos, en el Departamento Cesar, en las coordenadas entre los $9^{\circ} 56^{\prime} 14,84$ " $N$ y $73^{\circ} 51^{\prime} 46,72^{\prime \prime}$ W hasta los $9^{\circ} 55^{\prime} 14,12^{\prime \prime} \mathrm{N}$ y $73^{\circ} 51^{\prime} 5,78^{\prime \prime} \mathrm{W}$, a una altitud de $80 \mathrm{~m}$ s. n. m. En los suelos desnudos aledaños a la mencionada carretera en construcción fueron colectados entre 12 y 20 ejemplares de tres especies de gramíneas, bajo las siguientes condiciones de sustrato:

Especie 1. Condiciones extremas, en un suelo desnudo de vegetación con gravillas sin materia orgánica.

Especie 2. Condiciones extremas en un suelo con gravilla, arena y trazas de suelo fértil.

Especie 3. Un suelo desnudo de consistencia arenosa, pero con residuos de suelo fértil, que se había acumulado, supuestamente, por arrastres de las lluvias, donde también estaban presentes, en ocasiones, las especies 1 y 2 .

Las especies fueron colectadas en estado reproductivo, conservadas y herborizadas en el Herbario de la Facultad de Ciencias Básicas, de la Universidad de Pamplona, Colombia, donde fueron depositadas con los códigos: Leónides Castellanos 1, taxón 1, Leónides Castellanos 2, taxón 2, y Leónides Castellanos 3, taxón 3. Se aplicaron las claves de identificación y de descripciones morfológicas correspondientes, para llegar a género y especie, respectivamente (Valdés \& Allred, 2003; Sulekic, 2003; Giraldo-Cañas, 2011; 2013).

Se tomaron tres sub-muestras de las raíces y del suelo, de cada una de las muestras de plantas, en bolsas plásticas rotuladas y se llevaron a los laboratorios de Microbiología de la Facultad de Ciencias Básicas y al Centro de Investigaciones de Sanidad Vegetal y Bioinsumos (CISVEB), de la Facultad de Ciencias Agrarias, de la Universidad de Pamplona. En el laboratorio, se procesaron las raíces, para determinar las variables micorrízicas y se extrajeron muestras de raicillas de las submuestras, mencionadas anteriormente, tomando, para ello, los sistemas radicales y el suelo asociado, a una profundidad de hasta $10 \mathrm{~cm}$. Las raicillas menores de $2 \mathrm{~mm}$ de diámetro se lavaron y se cortaron en, aproximadamente, $1 \mathrm{~cm}$ de longitud y fueron teñidas con Azul de Tripán, según el método de Phillips \& Hayman (1970).
Las variables biológicas de las micorrizas en las raíces que se determinaron fueron: colonización micorrizica (Col \%) y presencia de arbúsculos y vesículas (porcentaje de raíces que presentaron estas estructuras).

La colonización micorrízica indica el nivel de presencia de hifas de HMA en las raicillas. Para su determinación, se utilizó la técnica del transecto lineal (\& Mosse, 1980), observándose en 100 intersecciones de las líneas verticales y horizontales de placa cuadriculada (1,27cm de separación). Se determinó, sobre las intercepciones, cuáles segmentos de raíces tenían presencia de hifas de HMA. En el mismo procedimiento, se contabilizaron raíces en los 100 interceptos, la presencia de arbúsculos y de vesículas, para estimar, posteriormente, el porcentaje.

Para determinar la concentración de esporas de los HMA, se tomaron $200 \mathrm{~g}$ del suelo de cada una de las muestras que acompañaba a las plantas de los tres taxones, en forma independiente y se homogeneizaron. De cada una, se tomaron $10 \mathrm{~g}$ de suelo y se depositaron en envases de $250 \mathrm{~mL}$ y luego se le añadieron $200 \mathrm{~mL}$ de agua destilada estéril y se agitaron. Las esporas de HMA se extrajeron de las muestras de suelo, empleando el método de decantado húmedo y tamizado, según protocolo descrito por Gerdemann \& Nicolson (1963), a través de tres tamices de 240,74 y $53 \mu \mathrm{m}$, respectivamente. El contenido obtenido en el último tamiz, se llevó a un tubo de ensayo, con la ayuda de un frasco lavador. Se centrifugó a 3000rpm, durante 3 min y se retiró el sobrenadante. Después, se le añadieron $50 \mathrm{~mL}$ de una solución de sacarosa al $75 \%$, con Tween 80 al $2 \%$ y se volvió a centrifugar, a 2000rpm, durante 2 min. El sobrenadante se pasó por un embudo con papel de filtro, se le añadió agua destilada para retirar la sacarosa y las esporas se contaron y se clasificaron bajo el estereoscopio, con un aumento 10X.

Se realizó un análisis estadístico no paramétrico de comparación de proporciones muestreales de la colonización micorrízica, el porcentaje de raíces con arbúsculos y el porcentaje de raíces con vesículas, entre las tres especies de gramíneas, para lo cual, se empleó el paquete estadístico Statistix versión 4 , con una probabilidad de error $\mathrm{P} \leq 0,01$.

\section{RESULTADOS Y DISCUSIÓN}

Las tres especies de gramíneas identificadas correspondieron a: $1=$ Aristida adscensionis L.; $2=$ Aristida ternipes Cav. y $3=$ Bothriochloa barbinodis (Lag.) Herter. El género Aristida comprende plantas anuales o perennes, xerófilas, que se reconocen fácilmente por el ápice de la lemma 3-aristado (Sulekic, 2003). Para Colombia, se informan 15 especies del género Aristida y seis, del género Bothriochloa (GiraldoCañas, 2011). Según este autor, las tres especies colectadas son nativas de Colombia: A. adscensionis, abunda entre 500 
y $2.500 \mathrm{~m}$ s. n. m.; A. ternipes, entre 0 y $1.000 \mathrm{~m} \mathrm{s.} \mathrm{n.} \mathrm{m.} y$ $B$. barbinodis, entre 0 y $2.000 \mathrm{~m} \mathrm{~s}$. n. m. Las dos especies de Aristida se informan para el departamento Cesar (región caribe), mientras que $B$. barbinodis, para los departamentos Tolima, Cundinamarca y Caldas (región andina), Cauca y Nariño (región pacífica). Otros autores, como Valdés \& Allred (2003), informan que A. adscensionis tiene su origen en la Isla Ascensión, ubicada en el Océano Atlántico, entre Brasil y África.
Las tres especies de gramíneas presentaron las raíces micorrizadas y con presencia de arbúsculos y vesículas grandes (Figuras 1a, 1b y 1c), aunque se detectaron diferentes porcentajes de colonización micorrízica, que varió entre 25 y 55\%. Asimismo, la presencia de arbúsculos osciló entre 20 y $40 \%$ y la de vesículas entre 10 y $20 \%$; sin embargo, el análisis de proporciones muestreales no arrojó diferencias estadísticas para estos parámetros, entre las tres especies de gramíneas (Tabla 1).

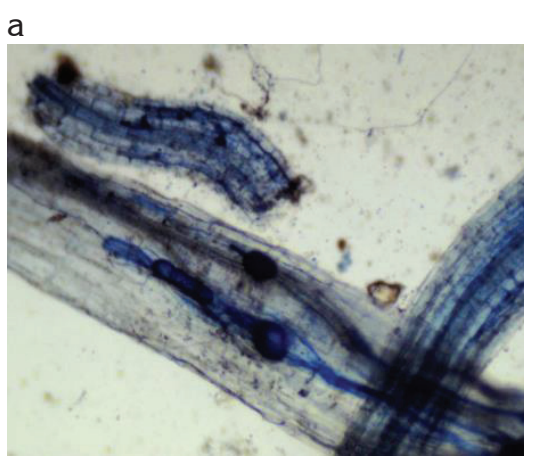

d

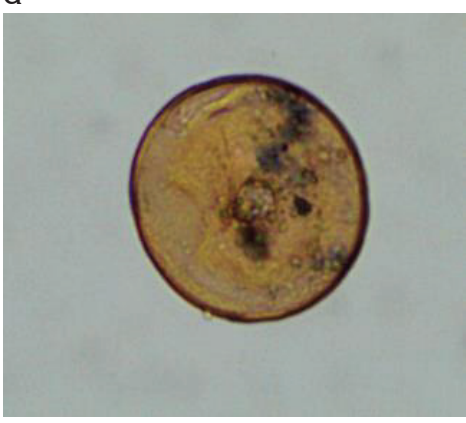

b

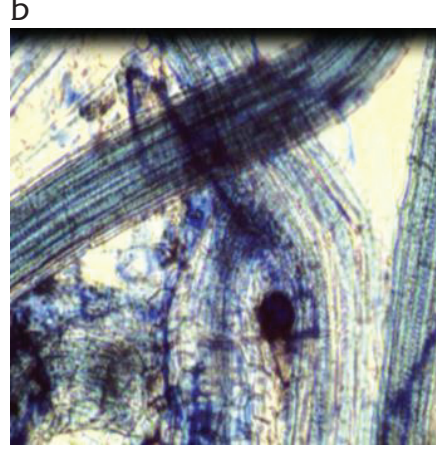

e

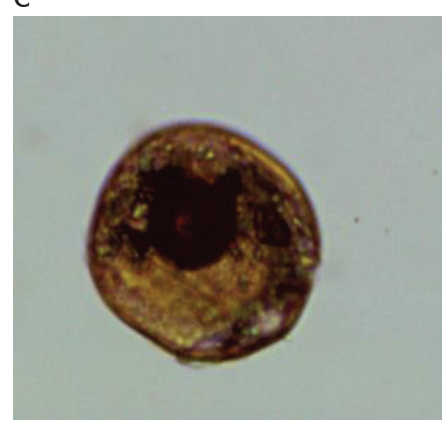

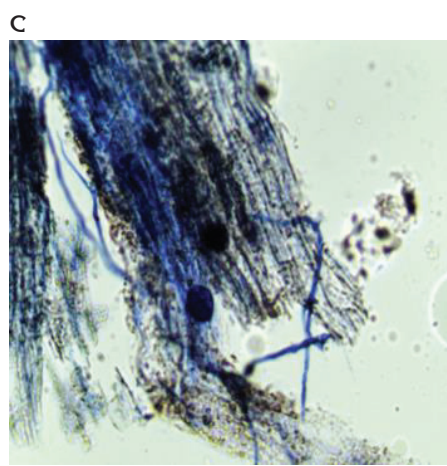

f

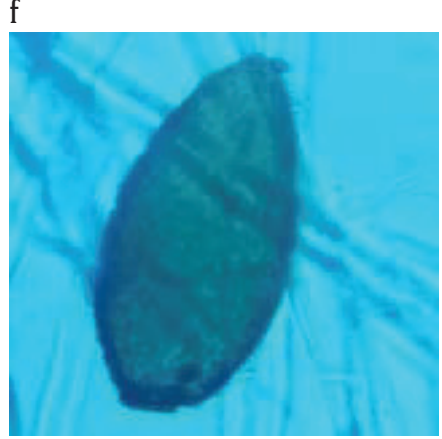

Figura 1. (a) Raíces micorrizadas de A. adscensionis; (b) A. ternipes y (c) B. barbinodis. Tipo de espora presente en el suelo de: (d) A. adscensionis; (e) A. ternipes y (f) B. barbinodis.

Tabla 1. Porcentaje de colonización micorrízica, presencia de arbúsculos y vesículas, en las raíces de cada especie de gramínea.

\begin{tabular}{|l|c|c|c|}
\hline $\begin{array}{c}\text { Especies de } \\
\text { gramíneas }\end{array}$ & $\begin{array}{c}\text { Colonización } \\
\text { micorrízica } \\
\mathbf{( \% )}\end{array}$ & $\begin{array}{c}\text { Raíces con } \\
\text { arbúsculos } \\
\mathbf{( \% )}\end{array}$ & $\begin{array}{c}\text { Raíces con } \\
\text { vesículas } \\
\mathbf{( \% )}\end{array}$ \\
\hline A. adscensionis & $33 \mathrm{~ns}$ & $25 \mathrm{~ns}$ & $10 \mathrm{~ns}$ \\
\hline A. ternipes & $55 \mathrm{~ns}$ & $40 \mathrm{~ns}$ & $20 \mathrm{~ns}$ \\
\hline B. barbinodis & $25 \mathrm{~ns}$ & $20 \mathrm{~ns}$ & $10 \mathrm{~ns}$ \\
\hline
\end{tabular}

ns: No significativo por la prueba de proporciones muestreales, $\mathrm{P} \leq 0,01$. 
Los niveles de colonización están en los rangos informados por Pérez et al. (2010), para Dichanthium aristatum (L.), en Colombia, pero superan los valores observados naturalmente en $B$. decumbens, tanto en el período lluvioso como poco lluvioso, en un estudio realizado en Cuba, tratamientos de experimentos, donde no se aplicó $\mathrm{P}_{2} \mathrm{O}_{5}$ (González et al. 2015).

Se detectaron esporas de HMA en los suelos rizosféricos de las tres especies de gramíneas, con valores que oscilaron entre 4,3 y 7,3 esporas por $g$ de suelo, en $A$. adscensionis y A. ternipes, respectivamente. Estos valores son menores que los encontrados en Brachiaria decumbens, por Posada et al. (2007), quienes cuantificaron valores de 15,74 y 23,75 esporas por $g$ de suelo, en dos zonas de Colombia.

Se presentaron una gran variedad de tipos de esporas de Glomeromycota en el suelo rizosférico de $A$. adscensionis y $A$. ternipes y solo tres en el de $B$. barbinodis. Aunque hay que profundizar en la identificación de las especies de HMA, algunas de las esporas asociadas a los suelos de las dos especies de Aristida corresponden con las características de Glomus (Figuras 1d y 1e), según morfología descrita por Hernández et al. (2014), redondeadas y más bien pequeñas, no así las de $B$. barbinodis, que eran de mayor tamaño y oblongas (Figura 1f).

Las conexiones subterráneas, mediante una red de hifas de hongos micorrízicos con las plantas, tanto a nivel intraespecífico como interespecífico, pone de manifiesto la potencial importancia en la absorción y el transporte de sustancias, cuya coexistencia de relaciones ecológicas y reproductivas son mantenidas por un largo periodo, favoreciendo un rápido crecimiento de las plantas y establecimiento de la regeneración (Azcón-Aguilera \& Barea, 2015). En la última década se ha enfatizado que para el desarrollo de programas de restauración ecológica es importante considerar la rizosfera, en particular, la aplicación de inoculantes micorrízicos, con énfasis sobre la protección de la microbiota indígena y la evaluación de las necesidades de inoculación.

Se hace necesario continuar los estudios para definir el potencial de estas gramíneas nativas para la restauración de la vegetación en las áreas aledañas a las carreteras, donde actualmente se emplea $B$. decumbens. Además, futuras investigaciones deberían permitir identificar los HMA y hacer pruebas de reproducción y de eficacia de estos hongos. Esta alternativa de restauración ecológica puede brindar resultados de éxito en el establecimiento de la vegetación, en áreas denudas y evitar problemas de erosión, con la consecuencia de impacto medioambiental no deseado.
Conflicto de intereses: El manuscrito fue preparado y revisado con la participación de todos los autores, quienes declaramos que no existe conflicto de interés, que ponga en riesgo la validez de los resultados presentados.

\section{BIBLIOGRAFÍA}

1. AZCÓN-AGUILAR, C.; BAREA, J.M. 2015. Nutrient cycling in the mycorrhizosphere. J. Soil Sci. Plant Nutr. 25:372-396.

2. DÍAZ, C. 2011. Alternativas para el control de la erosión mediante el uso de coberturas convencionales, no convencionales y revegetalización. Ingeniería e investigación. 31(3):80-90.

3. GERDEMANN, J.W.; NICOLSON, T.H. 1963. Spores of mycorrhizal. endogone species extracted from soil by wet sieving and decanting. Transact. British Mycol. Soc. 46:235-244.

4. GIOVANETTI, M.; MOSSE, B. 1980. An evaluation of techniques for measuring vesicular arbuscular mycorrhizal infection in roots. New Phytologist. 84:489-500.

5. GIRALDO-CAÑAS, D. 2013. Las gramíneas en Colombia. Riqueza, distribución, endemismo, invasión, usos y taxonomías populares. Universidad Nacional. Arfo Editores e Impresores Ltda. Bogotá. Colombia. 380p.

6. GIRALDO-CAÑAS, D. 2011. Catálogo de la familia Poaceae en Colombia. Darwiniana. 49(2):139-147.

7. GONZÁLEZ, P.J.; RAMÍREZ, J.F.; MORGAN, O.; RIVERA, R.; PLANA, R. 2015. Contribución de la inoculación micorrízica a la reducción de la fertilización fosfórica en Brachiaria decumbens. Cultivos Tropicales. (Cuba). 36(1):135-142.

8. HERNÁNDEZ, J.L.; SÁNCHEZ, C.L.; PALMA, F.J. 2014 Caracterización morfológica de micorriza arbuscular asociada a Agave potatorum Zucc. con potencial de uso agronómico. Rev. Mex. Agroecosistemas. 1(2):82-93.

9. KJOLLER, A.; MULLER, M.; SSTRUWE, A.; VOLTERS, V.; PLUG, A. 2000. Diversity and role of microorganisms. En: Schulze E.D. (ed.). Carbon and nitrogen cycling in European forest ecosystems. Springer-Verlag., Berlin, Germany. p.382-402.

10. MEDINA, A.; AZCÓN, R. 2010. Effectiveness of the application of arbuscular mycorrhizal fungi and organic 
amendments to improve soil quality and plant performance under stress conditions. J. Soil Sci. Plant Nutr. 10(3):354-372.

11. MINISTERIO DE DESARROLLO ECONÓMICO. 2002. Viceministerio de Transporte, Comunicación y Aeronáutica Civil. Programa para la conservación de la red vial fundamental con microempresas y administradores. Disponible desde Internet en: http:// zietlow.com/docs/ManualTMEBol2.pdf (con acceso 20/12/2017.

12. NUFARM COLOMBIA. 2016. Brachiaria decumbens. Disponible desde Internet en: http://www.nufarm.com/ CO/BrachiariaDecumbens (con acceso 22/10/2016).

13. PÉREZ, A.; ROJAS, J.; FUENTES, J. 2010. Determinación de un modelo logístico para evaluación in situ de la colonización de micorrizas en pasto Dichanthium aristatum (L.). Rev. Col. Cienc. Anim. 2(1):7384.

14. PHILLIPS, J.M.; HAYMAN, D.S. 1970 Improved Procedures for Clearing Roots and Staining Parasitic Vesicular-Arbuscular Mycorrhizal Fungi for Rapid Assessment of Infection. Transact. British Mycol. Soc. 55:158-161.

15. POSADA, R.H.; FRANCO L.A.; CUÉLLAR, A.P.; SÁNCHEZ, W.; SÁNCHEZ, A.P. 2007. Inóculo de hongo de micorriza arbuscular en pasturas de Brachiaria decumbens (Poaceae) en zonas de loma y vega. Acta Biol. Col. 12(1) :113-119.

16. SALDIAS, M.G. 2011. Jardinería en Chile. Ediciones Universidad Central. (Chile). 258p.

17. SULEKIC, A.A. 2003. Revisión de las especies del género Aristida (Poaceae, Aristideae) del noroeste de la Argentina. Darwiniana 41(1-4):155-188.

18. TÁCUNA, R.E.; AGUIRRE, L.; FLORES, E.R. 2015. Influencia de la revegetación con especies nativas y la incorporación de materia orgánica en la recuperación de pastizales degradados. Ecología Aplicada. 14(2):191-200.

19. TORMO, J.; BOCHET, E.; GARCÍA-FAYOS, P. 2009. Restauración y revegetación de taludes de carreteras en ambientes mediterráneos semiáridos: procesos edáficos determinantes para el éxito Ecosistemas. 18(2):79-90.

20. VALDÉS, J.; ALLRED, K.W. 2003. El género Aristida (Gramineae) en el noreste de México. Acta Botánica Mexicana. 63:1-45.

Recibido: Agosto 19 de 2017

Aceptado: Marzo 29 de 2018

Cómo citar:

Castellanos González, L.; Forero Cuadros, J.; Rodríguez Rincón, F.; Luis Roberto Sánchez Montano, L.R. 2018. Gramíneas nativas en suelos desnudos al borde de una carretera y su simbiosis con micorrizas. Rev. U.D.C.A Act. \& Div. Cient. 21(1): 253-257. 